\title{
A Search-based Neural Model for Biomedical Nested and Overlapping Event Detection
}

\author{
Kurt Espinosa $^{1,4}$, Makoto Miwa ${ }^{2,3}$, Sophia Ananiadou ${ }^{1}$ \\ ${ }^{1}$ National Centre for Text Mining, School of Computer Science, The University of Manchester, UK \\ ${ }^{2}$ Toyota Technological Institute, Nagoya, 468-8511, Japan \\ ${ }^{3}$ Artificial Intelligence Research Center (AIRC), \\ National Institute of Advanced Industrial Science and Technology (AIST), Japan \\ ${ }^{4}$ Department of Computer Science, University of the Philippines Cebu, Cebu City, Philippines \\ \{kurtjunshean.espinosa, sophia.ananiadou\}@manchester.ac.uk \\ makoto-miwaltoyota-ti.ac.jp
}

\begin{abstract}
We tackle the nested and overlapping event detection task and propose a novel search-based neural network (SBNN) structured prediction model that treats the task as a search problem on a relation graph of trigger-argument structures. Unlike existing structured prediction tasks such as dependency parsing, the task targets to detect DAG structures, which constitute events, from the relation graph. We define actions to construct events and use all the beams in a beam search to detect all event structures that may be overlapping and nested. The search process constructs events in a bottom-up manner while modelling the global properties for nested and overlapping structures simultaneously using neural networks. We show that the model achieves performance comparable to the state-of-the-art model Turku Event Extraction System (TEES) on the BioNLP Cancer Genetics (CG) Shared Task 2013 without the use of any syntactic and hand-engineered features. Further analyses on the development set show that our model is more computationally efficient while yielding higher F1-score performance.
\end{abstract}

\section{Introduction}

Nested and overlapping event structures, which occur widely in text, are important because they can capture relations between events such as causality, e.g., a "production" event is a consequence of a "discovery" event, which in turn is a result of an "exploration" event. Event extraction involves the identification of a trigger and a set of its arguments in a given text. Figure 1 shows an example of a nested and overlapping event structure in the biomedical domain. The relation graph (topmost) forms a directed acyclic graph (DAG) structure (McClosky et al., 2011) and it encapsulates 15 event structures. It contains nested event

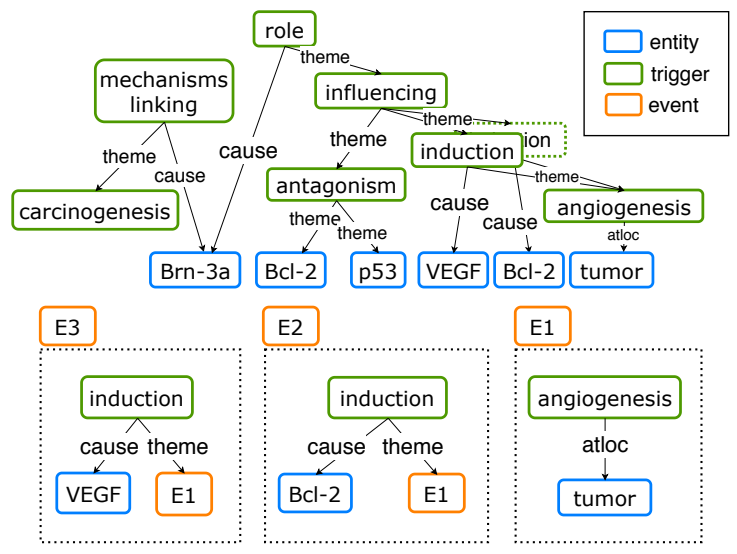

Figure 1: Top: A DAG-structured relation graph (topmost) from the sentence "Looking for mechanisms linking Brn-3a to carcinogenesis, we discuss the role of this transcription factor in influencing Bcl-2/VEGF induction of tumor angiogenesis, ..." from BioNLP'13 CG Shared Task (Pyysalo et al., 2015). Bottom: A pair of overlapping and nested events $(E 2, E 3)$ extracted from the graph with their shared argument event, a flat event $(E 1)$.

structures such as $E 2, E 3$ because one of their arguments, in this case $E 1$, is an event. Specifically, $E 1$ is a flat event since its argument is an entity. Moreover, E2 and E3 are also overlapping events (explicitly shown in the relation graph having two induction triggers) because they share a common argument, E1.

State-of-the-art approaches to event extraction in the biomedical domain are pipeline systems (Björne and Salakoski, 2018; Miwa et al., 2013) that decompose event extraction into simpler tasks such as: i) trigger/entity detection, which determines which words and phrases in a sentence potentially constitute as participants of an event, ii) relation detection, which finds pairwise relations between triggers and arguments, and iii) event detection, which combines pairwise 
relations into complete event structures. Joint approaches have also been explored (Rao et al., 2017; Riedel and McCallum, 2011; Vlachos and Craven, 2012; Venugopal et al., 2014), but they focus on finding relation graphs and detect events with rules. McClosky et al. (2011) treats events as dependency structures by constraining event structures to map to trees, thus their method cannot represent overlapping event structures. Other neural models in event extraction are in the general domain (Feng et al., 2016; Nguyen and Grishman, 2015; Chen et al., 2015; Nguyen et al., 2016), but they used the ACE2005 corpus which does not have nested events (Miwa et al., 2014). Furthermore, there are some efforts on applying transition-based methods on DAG structures in dependency parsing, e.g., (Sagae and Tsujii, 2008; Wang et al., 2018), however, they do not consider overlapping and nested structures.

We present a novel search-based neural event detection model that detects overlapping and nested events with beam search by formulating it as a structured prediction task for DAG structures. Given a relation graph of trigger-argument relations, our model detects nested events by searching a sequence of actions that construct event structures incrementally in a bottom-up manner. We focus on event detection since the existing methods do not consider nested and overlapping structures as a whole during learning. Treating them simultaneously helps the model avoid inferring wrong causality relations between entities. Our model detects overlapping events by maintaining multiple beams and detecting events from all the beams, in contrast to existing transitionbased methods (Nivre, 2003, 2006; Chen and Manning, 2014; Dyer et al., 2015; Andor et al., 2016; Vlachos and Craven, 2012). We define an LSTM-based neural network model that can represent nested event structure to choose actions.

We show that our event detection model achieves performance comparable to the event detection module of the state-of-the-art TEES system (Björne and Salakoski, 2018) on the BioNLP CG Shared Task 2013 (Pyysalo et al., 2013) without the use of any syntactic and hand-engineered features. Furthermore, analyses on the development set show our model performs fewer number of classifications in less time.

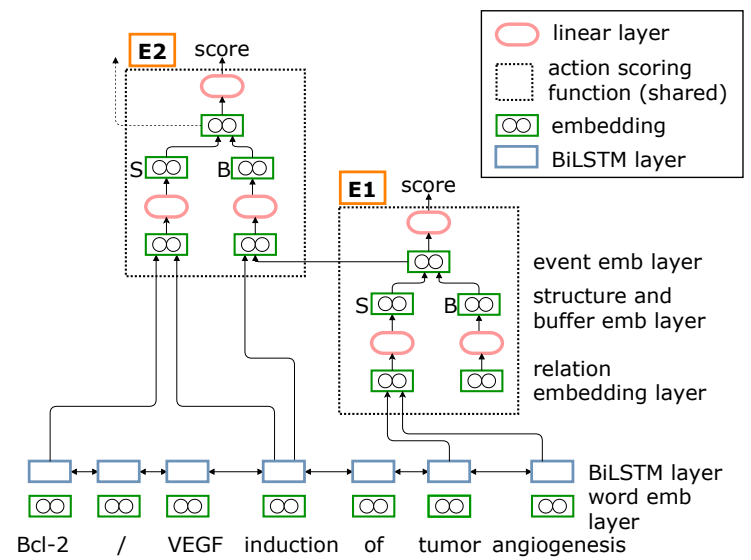

Figure 2: An illustration of the proposed neural model detecting event structures in a bottom-up manner, where E1 event representation becomes an argument to E2 event structure on the example sentence used in Figure 1.

\section{Model}

We describe our search-based neural network (SBNN) model that constitutes events from a relation graph by structured prediction. SBNN resembles an incremental transition-based parser (Nivre, 2006), but the search order, actions and representations are defined for DAG structures. We first discuss how we generate the relation graph in $\S 2.1$, then describe the structured prediction algorithm in $\S 2.2$ and the neural network in $\S 2.3$ and lastly, we explain the training procedure in $\S 2.4$.

\subsection{Relation Graph Generation}

To train our model, we use the predicted relations merged with pairwise relations decomposed from the gold events. We then generate a relation graph from the merged relations. During inference, the relation graph is generated only from the predicted relations. Figure 1 shows an example of the generated DAG-structured relation graph.

\subsection{Structured Prediction for DAGs}

We represent event structures with DAG structures and find them in a relation graph. Our model performs beam search on relation graphs by choosing actions to construct events. Unlike existing beam search usage where they only choose the best path in the beam, e.g., (Nivre, 2006), we use all the beams to predict event structures, which enables the model to predict overlapping and nested events.

Event structures are searched and fixed for each 


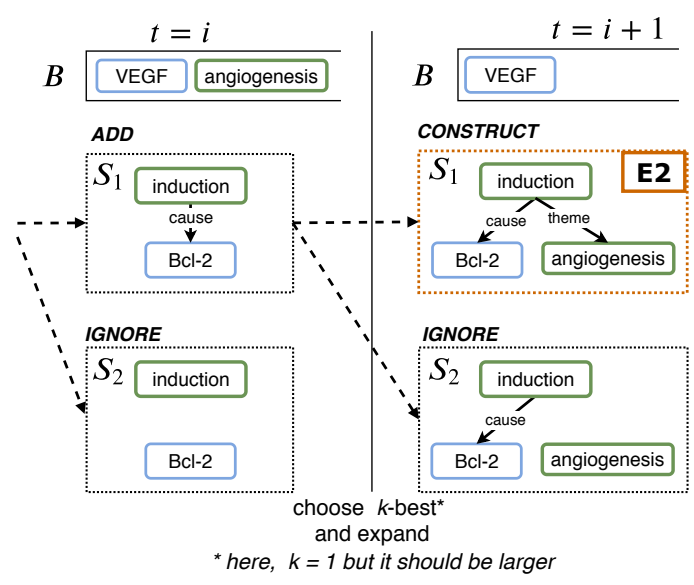

Figure 3: A snapshot of the search procedure within one time step (with $k=1$ ) on the trigger induction which detects event $E 2$ after a CONSTRUCT action.

trigger of the relation graph in a bottom-up manner. The model predicts the flat events first and then the representations of the flat events become the argument of the nested events. Figure 2 shows the proposed neural model (described in detail in $\S 2.3$ ), which illustrates that the event representation of event $E 1$ becomes the argument to event $E 2$. If the flat event is not detected, its nested event will not be detected consequently. When this happens, the search process stops.

Figure 3 shows an snapshot of the search procedure within one time step as applied to a relation graph with a trigger induction to detect event $E 2$ (see Figure 1 and 2). To do the search, the model maintains two data structures: a buffer $B$ which holds a queue of arguments ${ }^{1}$ to be processed and a structure $S$ which contains the partially built event structure. The initial state is composed of the buffer $B$ with all the arguments and a structure $S$ empty. At each succeeding time step, the model applies a set of predefined actions to each argument and uses a neural network to score those actions. Processing completes when $B$ is empty and $S$ contains all the arguments for the trigger along with the history of actions taken by the model.

We now define the three actions ${ }^{2}$ that the model applies at each time step to each argument, namely: add the argument (ADD), ignore the argument (IGNORE) and add the argument and construct an event candidate (CONSTRUCT).

\footnotetext{
${ }^{1}$ A special NONE argument marks the first argument in the buffer to enable detection of no-argument events.

${ }^{2}$ Except for the NONE argument where only two actions are applied: IGNORE and CONSTRUCT.
}

We have chosen only three actions for simplicity. Figure 3 shows how actions are applied to the argument angiogenesis (due to space constraints we only show two actions indicated by the arrows). Concretely, we show two distinct structures $\left(S_{1}, S_{2}\right)$ that are created in time step $t+1$ after CONSTRUCT and IGNORE actions were applied to the current structure $S_{1}$ in time step $i$.

The event candidate structures are fixed as events if the scores of the CONSTRUCT actions are above a certain threshold. The resulting state after a CONSTRUCT action is removed from the beams. We maintain multiple beams and use all of them to predict overlapping events.

\subsection{Neural Network as Scoring Function}

Figure 2 shows the proposed neural model. We employ a BiLSTM network to generate the word representations from pre-trained word embeddings. To represent phrases, we averaged the word representations. The LSTM network is shared among the states during search in the sentence. We build a relation embedding for each argument, which concatenates the information of the trigger $t$, the role $o$, the argument $a$ and the action $c$. We include both the type information $p$ and the word or phrase representation $w$ of the trigger or entity argument. Formally, each relation $r_{i}$ is represented as a relation embedding: $\mathbf{r}_{i}=$ $\left[\mathbf{t}_{p} ; \mathbf{t}_{w} ; \mathbf{o}_{p} ; \mathbf{a}_{p} ; \mathbf{a}_{w} ; \mathbf{c}\right]$, where $\mathbf{t}_{p}$ is the representation of the type of the trigger and so on. Each $\mathbf{r}_{i}$ is passed to a linear hidden layer, then to a rectified linear unit (ReLU) non-linearity and summed to produce the structure and buffer embeddings: $\mathbf{S}_{t}$ and $\mathbf{B}_{t}$.

We use a neural network as the action scoring function (indicated by the dotted box in Figure 2) defined as $\sigma\left(a_{t} \mid S_{t-1}, B_{t-1}\right)$. We model the action scoring function using $S_{t}$ and $B_{t}$, which are composed by adding an action $a_{t}$ for a relation $r_{t}$ to $S_{t-1}$ and moving $r_{t}$ from $B_{t-1}$ to $S_{t-1}$. The state at any time step $t$ is composed of the buffer $B_{t}$ and the partially built structure $S_{t}$. Each of $S_{t}$ and $B_{t}$ contains a set of relations $\left\{r_{1}, r_{2}, r_{3}, \ldots, r_{n}\right\}$. In Figure 2, there is no arrow to $B$ in event $E 1$ because the diagram only shows the model snapshot at a specific time step during the search process. In this particular time step $t$, the buffer $B$ in $E 1$ is already empty and thus, it does not contain any relations $\mathbf{r}_{i}$.

$\mathbf{S}_{t}$ and $\mathbf{B}_{t}$ are then concatenated to form the 
event embedding. The event embedding has the same dimension as the sum of argument type and word dimensions so that it can be used as argument representation in nested events as shown in Figure 2. Then, we passed the event embedding into a linear hidden layer and output $z_{t}$. Finally, the scoring function $\sigma$ is calculated as $\sigma\left(a_{t} \mid S_{t-1}, B_{t-1}\right)=\operatorname{sigmoid}\left(z_{t}\right)$.

\subsection{Training}

From the relation graph generated in $\S 2.1$, we calculate gold action sequences that construct the gold event structures on the graph. The loss is summed over all actions and for all the events during the beam search and thus the objective function is to minimise their negative log-likelihood. We employ early updates (Collins and Roark, 2004): if the gold falls out of the beam, we stop searching and update the model immediately.

\section{Experimental Settings}

We applied our model to the BioNLP CG shared task 2013 (Pyysalo et al., 2015). We used the original data partition and employed the official evaluation metrics. We focussed on the CG task dataset over other BioNLP datasets because of its complexity and size (Nédellec et al., 2013; Björne and Salakoski, 2018; Pyysalo et al., 2013). The CG dataset has the most number of entity types and event types and thus is the most complex among the available (and accessible) BioNLP datasets. Furthermore, the CG dataset is the largest dataset in terms of the number of event instances and the proportion of nested and overlapping events. Evaluating our model extensively on other BioNLP tasks and datasets is part of our future work.

The development set contains 3,217 events of which $36.46 \%$ are nested events, $43.05 \%$ are overlapping events and $44.07 \%$ are flat events. Note that the total does not equal to $100 \%$ because nested and overlapping events may have intersection: a nested event can be overlapping and vice versa.

We compared our model with the event detection module of the state-of-the-art model TEES (Björne and Salakoski, 2018), which employs convolutional neural network and uses syntactic and hand-engineered features for event detection. Björne and Salakoski (2018) found that the dependency parse features increased the performance of the convolutional model. In con- trast, we do not use these syntactic features nor hand-engineered features. Furthermore, instead of the ensemble methods, we used TEES's published single models, as this enables us to make a direct comparison with TEES in a minimal setting. We train our model using the predicted relations from TEES merged with the pairwise relations decomposed from the gold CG events. During inference, we predict event structures using only the predicted relations from TEES.

\subsection{Nested and Overlapping Event Evaluation Process}

Similarly, we used the official evaluation script to measure the performance of the model on nested, overlapping and flat events. We first separated the nested, overlapping and flat events, respectively. Then we compute the precision and recall for each category in the following way. For example, for nested events, to compute the precision, we compare the predicted nested events with all gold events and to compute recall, we compare gold nested events with all predicted events. The evaluation script detects nested events by comparing the whole tree structure down to its sub-events until it reaches the flat events. Hence, the performance scores of the nested events inevitably include the performance on flat events.

\subsection{Training Details and Model Parameters}

We implemented our model using the Chainer library (Tokui et al., 2015). We initialised the word embeddings using pre-trained embeddings (Chiu et al., 2016) while other embeddings are initialised using the normal distribution. All the embeddings and weight parameters were updated with mini-batch using the AMSGrad optimiser (Reddi et al., 2018). We also incorporated early stopping to choose the number of training epochs and tuned hyper-parameters (dropout, learning rate and weight decay rate) using grid search. The model parameters can be found in appendix A.

\section{Results and Analyses}

Table 1 shows the event detection performance of the models on the test set. Our model achieves performance comparable to the state-of-the-art TEES event detection module without the use of any syntactic and hand-engineered features, suggesting it can be applied to other domains with no need for feature engineering. We validated it to have no sig- 


\begin{tabular}{llcc}
\hline Model & $\mathrm{P}$ & $\mathrm{R}$ & $\mathrm{F} 1(\%)$ \\
\hline TEES & 61.42 & $\mathbf{5 2 . 9 3}$ & 56.86 \\
SBNN & $\mathbf{6 3 . 6 7}$ & 51.43 & $\mathbf{5 6 . 9 0}$ \\
\hline
\end{tabular}

Table 1: Event detection performance on the CG task 2013 test dataset.

\begin{tabular}{llcc}
\hline Model & $\mathrm{P}$ & $\mathrm{R}$ & $\mathrm{F}(\%)$ \\
\hline TEES & 56.81 & 48.21 & 52.16 \\
\hline SBNN $k=1$ & 48.95 & 43.79 & 46.23 \\
$k=8$ & $\mathbf{6 3 . 6 0}$ & 47.46 & $\mathbf{5 4 . 3 6}$ \\
$k=64$ & 60.30 & $\mathbf{4 9 . 1 6}$ & 54.17 \\
$k=256$ & 60.91 & 48.53 & 54.02 \\
\hline
\end{tabular}

Table 2: Event detection performance on CG task 2013 development set for SBNN with the top three performing $k$-values and when $k=1$.

nificant statistical difference with the TEES model (the Approximate Randomisation test (Yeh, 2000; Noreen, 1989)).

To gain a deeper insight about the model, we performed analyses on the development set. Table 2 shows the performance of SBNN by varying the $k$-best parameter in beam search. We tested $2^{i}$ values for $i=1,2,3, \ldots, 11$ and found that the best value was 8 with F1-score of $54.36 \%$, which is 2.2 percentage points (pp) higher than TEES.

Table 3 shows the number of classifications (or action scoring function calls in our model) performed by each model with the corresponding actual running time. SBNN performs fewer classifications and in less time than TEES, implying it is more computationally efficient.

Table 4 shows the performance comparison of the models on nested, overlapping and flat event detection. Our model yields higher F1-scores than TEES which can be attributed to its ability to maintain multiple beams and to detect events from all these beams during search.

Finally, we computed the upper bound recall given the predicted relations from TEES. The upper bound is computed by setting the threshold parameter of our model to zero, which then constructs all gold events possible from the predicted relations of TEES. Since we evaluate our model on the output relations of TEES, the event detection performance is bounded or limited by these predicted relations. For instance, if one of the relations in an event was not predicted, the event structure will never be formed. We observe that this remains a challenging task since the upper bound recall is still at $53.47 \%$ (6.01pp higher than our

\begin{tabular}{lrr}
\hline Model & Number of Classifications & Running Time (s) \\
\hline TEES & 6,141 & 155 \\
SBNN $k=8$ & $\mathbf{4 , 0 9 3}$ & $\mathbf{1 3 1}$ \\
\hline
\end{tabular}

Table 3: Comparison on computational efficiency on the CG task 2013 development dataset.

\begin{tabular}{lcccc}
\hline Model & Nested & Overlapping & Flat & Overall F1 (\%) \\
\hline TEES & 42.70 & 34.49 & 56.81 & 52.16 \\
SBNN $k=8$ & $\mathbf{4 5 . 2 4}$ & $\mathbf{3 6 . 9 2}$ & $\mathbf{6 0 . 5}$ & $\mathbf{5 4 . 3 6}$ \\
\hline
\end{tabular}

Table 4: Nested and overlapping event detection F1 (\%) score performance on the CG task 2013 development set.

current model's score). Closing this gap requires among others addressing inter-sentence and selfreferential events, which account for $3.1 \%$ of the total events.

\section{Conclusions and Future Work}

We presented a novel search-based neural model for nested and overlapping event detection by treating the task as structured prediction for DAGs. Our model achieves performance comparable to the state-of-the-art TEES event detection model without the use of any syntactic and hand-engineered features, suggesting the domainindependence of the model. Further analyses on the development set revealed some desirable characteristics of the model such as its computational efficiency while yielding higher F1-score performance. These results set the first focussed benchmark of our model and next steps include applying it to other event datasets in the biomedical and general domain. In addition, it can also be applied to other DAG structures such as nested/discontiguous entities (Muis and Lu, 2016; Ju et al., 2018).

\section{Acknowledgement}

We thank our anonymous reviewers for their invaluable feedback. This research was supported by funding from BBSRC Japan Partnering Award, Text mining and bioinformatics platforms for metabolic pathway modelling [Grant ID: BB/P025684/1] and AIRC/AIST. The first author gratefully acknowledges financial support from the University of the Philippines System Doctoral Studies Fund. 


\section{References}

Daniel Andor, Chris Alberti, David Weiss, Aliaksei Severyn, Alessandro Presta, Kuzman Ganchev, Slav Petrov, and Michael Collins. 2016. Globally normalized transition-based neural networks. In Proceedings of the 54th Annual Meeting of the Association for Computational Linguistics (Volume 1: Long Papers), pages 2442-2452, Berlin, Germany. Association for Computational Linguistics.

Jari Björne and Tapio Salakoski. 2018. Biomedical event extraction using convolutional neural networks and dependency parsing. In Proceedings of the BioNLP 2018 workshop, pages 98-108, Melbourne, Australia. Association for Computational Linguistics.

Danqi Chen and Christopher Manning. 2014. A fast and accurate dependency parser using neural networks. In Proceedings of the 2014 Conference on Empirical Methods in Natural Language Processing (EMNLP), pages 740-750, Doha, Qatar. Association for Computational Linguistics.

Yubo Chen, Liheng $\mathrm{Xu}$, Kang Liu, Daojian Zeng, and Jun Zhao. 2015. Event extraction via dynamic multi-pooling convolutional neural networks. In Proceedings of the 53rd Annual Meeting of the Association for Computational Linguistics and the 7th International Joint Conference on Natural Language Processing (Volume 1: Long Papers), pages 167-176, Beijing, China. Association for Computational Linguistics.

Billy Chiu, Gamal Crichton, Anna Korhonen, and Sampo Pyysalo. 2016. How to train good word embeddings for biomedical nlp. In Proceedings of the 15th Workshop on Biomedical Natural Language Processing, pages 166-174, Berlin, Germany. Association for Computational Linguistics.

Michael Collins and Brian Roark. 2004. Incremental parsing with the perceptron algorithm. In Proceedings of the 42nd Meeting of the Association for Computational Linguistics (ACL'04), Main Volume, pages 111-118, Barcelona, Spain.

Chris Dyer, Miguel Ballesteros, Wang Ling, Austin Matthews, and Noah A. Smith. 2015. Transitionbased dependency parsing with stack long shortterm memory. In Proceedings of the 53rd Annual Meeting of the Association for Computational Linguistics and the 7th International Joint Conference on Natural Language Processing (Volume 1: Long Papers), pages 334-343, Beijing, China. Association for Computational Linguistics.

Xiaocheng Feng, Lifu Huang, Duyu Tang, Heng Ji, Bing Qin, and Ting Liu. 2016. A languageindependent neural network for event detection. In Proceedings of the 54th Annual Meeting of the Association for Computational Linguistics (Volume 2: Short Papers), pages 66-71, Berlin, Germany. Association for Computational Linguistics.
Meizhi Ju, Makoto Miwa, and Sophia Ananiadou. 2018. A neural layered model for nested named entity recognition. In Proceedings of the 2018 Conference of the North American Chapter of the Association for Computational Linguistics: Human Language Technologies, Volume 1 (Long Papers), pages 1446-1459, New Orleans, Louisiana. Association for Computational Linguistics.

David McClosky, Mihai Surdeanu, and Christopher Manning. 2011. Event extraction as dependency parsing. In Proceedings of the 49th Annual Meeting of the Association for Computational Linguistics: Human Language Technologies, pages 16261635, Portland, Oregon, USA. Association for Computational Linguistics.

Makoto Miwa, Sampo Pyysalo, Tomoko Ohta, and Sophia Ananiadou. 2013. Wide coverage biomedical event extraction using multiple partially overlapping corpora. BMC bioinformatics, 14(1):175.

Makoto Miwa, Paul Thompson, Ioannis Korkontzelos, and Sophia Ananiadou. 2014. Comparable study of event extraction in newswire and biomedical domains. In Proceedings of COLING 2014, the 25th International Conference on Computational Linguistics: Technical Papers, pages 2270-2279, Dublin, Ireland. Dublin City University and Association for Computational Linguistics.

Aldrian Obaja Muis and Wei Lu. 2016. Learning to recognize discontiguous entities. In Proceedings of the 2016 Conference on Empirical Methods in Natural Language Processing, pages 75-84, Austin, Texas. Association for Computational Linguistics.

Claire Nédellec, Robert Bossy, Jin-Dong Kim, JungJae Kim, Tomoko Ohta, Sampo Pyysalo, and Pierre Zweigenbaum. 2013. Overview of bionlp shared task 2013. In Proceedings of the BioNLP Shared Task 2013 Workshop, pages 1-7.

Thien Huu Nguyen, Kyunghyun Cho, and Ralph Grishman. 2016. Joint event extraction via recurrent neural networks. In Proceedings of the 2016 Conference of the North American Chapter of the Association for Computational Linguistics: Human Language Technologies, pages 300-309, San Diego, California. Association for Computational Linguistics.

Thien Huu Nguyen and Ralph Grishman. 2015. Event detection and domain adaptation with convolutional neural networks. In Proceedings of the 53rd Annual Meeting of the Association for Computational Linguistics and the 7th International Joint Conference on Natural Language Processing (Volume 2: Short Papers), pages 365-371, Beijing, China. Association for Computational Linguistics.

Joakim Nivre. 2003. An efficient algorithm for projective dependency parsing. In Proceedings of the 8th International Workshop on Parsing Technologies (IWPT), pages 149-160, Nancy, France. 
Joakim Nivre. 2006. Inductive dependency parsing, 1 edition, volume 34 of Text, Speech and Language Technology. Springer Netherlands.

Eric W Noreen. 1989. Computer-intensive methods for testing hypotheses. Wiley New York.

Sampo Pyysalo, Tomoko Ohta, and Sophia Ananiadou. 2013. Overview of the cancer genetics (cg) task of bionlp shared task 2013. In Proceedings of the BioNLP Shared Task 2013 Workshop, pages 58-66.

Sampo Pyysalo, Tomoko Ohta, Rafal Rak, Andrew Rowley, Hong-Woo Chun, Sung-Jae Jung, Sung-Pil Choi, Jun'ichi Tsujii, and Sophia Ananiadou. 2015. Overview of the cancer genetics and pathway curation tasks of bionlp shared task 2013. BMC bioinformatics, 16(10):S2.

Sudha Rao, Daniel Marcu, Kevin Knight, and Hal Daumé III. 2017. Biomedical event extraction using abstract meaning representation. In BioNLP 2017, pages 126-135, Vancouver, Canada,. Association for Computational Linguistics.

Sashank J. Reddi, Satyen Kale, and Sanjiv Kumar. 2018. On the convergence of adam and beyond. In International Conference on Learning Representations.

Sebastian Riedel and Andrew McCallum. 2011. Robust biomedical event extraction with dual decomposition and minimal domain adaptation. In Proceedings of BioNLP Shared Task 2011 Workshop, pages 46-50, Portland, Oregon, USA. Association for Computational Linguistics.

Kenji Sagae and Jun'ichi Tsujii. 2008. Shift-reduce dependency dag parsing. In Proceedings of the 22nd International Conference on Computational Linguistics (Coling 2008), pages 753-760. Coling 2008 Organizing Committee.

Seiya Tokui, Kenta Oono, Shohei Hido, and Justin Clayton. 2015. Chainer: a next-generation open source framework for deep learning. In Proceedings of Workshop on Machine Learning Systems (LearningSys) in The Twenty-ninth Annual Conference on Neural Information Processing Systems (NIPS).

Deepak Venugopal, Chen Chen, Vibhav Gogate, and Vincent Ng. 2014. Relieving the computational bottleneck: Joint inference for event extraction with high-dimensional features. In Proceedings of the 2014 Conference on Empirical Methods in Natural Language Processing (EMNLP), pages 831-843, Doha, Qatar. Association for Computational Linguistics.

Andreas Vlachos and Mark Craven. 2012. Biomedical event extraction from abstracts and full papers using search-based structured prediction. BMC bioinformatics, 13(11):S5.
Yuxuan Wang, Wanxiang Che, Jiang Guo, and Ting Liu. 2018. A neural transition-based approach for semantic dependency graph parsing. In AAAI Conference on Artificial Intelligence.

Alexander Yeh. 2000. More accurate tests for the statistical significance of result differences. In Proceedings of the 18th conference on Computational linguistics-Volume 2, pages 947-953. Association for Computational Linguistics. 


\section{A Model Parameters}

\begin{tabular}{lr}
\hline Parameter & Value/Dimension \\
\hline Mini-Batch Size & 100 \\
Word Embedding & 200 \\
BiLSTM Word Embedding & 100 \\
Role Type Embedding & 10 \\
Trigger/Argument Type Embedding & 20 \\
Early Stopping Patience & 5 \\
Dropout & 0.5 \\
Learning Rate & 0.001 \\
Hidden Layer Size & 60 \\
Event Embedding & 100 \\
Action Score Threshold & 0.5 \\
Beam Size & 8 \\
Action Embedding & 4 \\
Weight Decay Rate & 0.001 \\
\hline
\end{tabular}

Table 5: Hyper-parameters used in our experiments. 